\title{
Wind accretion in symbiotic X-ray binaries
}

\section{K. Postnov ${ }^{* a}$, N. Shakura ${ }^{a}$, A. González-Galán ${ }^{b}$, E. Kuulkers ${ }^{c}$, P. Kretschmar ${ }^{c}$, S. Larsson $^{d}$, M.H. Finger ${ }^{e, f}$, A. Kochetkova ${ }^{a}$, G. Lü ${ }^{g}$ and L. Yungelson ${ }^{h}$}

${ }^{a}$ Sternberg Astronomical Institute, 13, Universitetskij pr., 119992 Moscow, Russia

${ }^{b}$ Departamento de Física, Ingeniería de Sistemas y Teoría de la Señal,Universidad de Alicante, Apdo. 99, 03080 Alicante, Spain

${ }^{c}$ European Space Astronomy Centre (ESAC), P.O. Box 78, 28691, Villanueva de la Cañada, Spain

${ }^{d}$ Department of Astronomy, Stockholm University, SE-106 91 Stockholm, Sweden

${ }^{e}$ National Space Science and Technology Center, 320 Sparkman Drive, Huntsville, AL 35805, USA

${ }^{f}$ Universities Space Research Association, 6767 Old Madison Pike, Suite 450, Huntsville, AL 35806, USA

${ }^{g}$ School of Physics, Xinjiang University, Urumqi, 830046 China

${ }^{h}$ Institute of Astronomy RAS, Moscow, 48 Pyatnitskaya Str., Moscow, 119017, Russia

E-mail: kpostnov@gmail.com, nikolai.shakura@gmail.com, anagonzalez@ua.es,Erik.Kuulkers@sciops.esa.int

Peter.Kretschmarlesa.int, stefandastro.su.se, mark.finger@nasa.gov, sunny@sai.msu.ru, guolianglvesina.com, lry@inasan.ru

The properties of wind accretion in symbiotic X-ray binaries (SyXBs) consisting of red-giant and magnetized neutron star (NS) are discussed. The spin-up/spin-down torques applied to NS are derived based on a hydrodynamic theory of quasi-spherical accretion onto magnetized NSs. In this model, a settling subsonic accretion proceeds through a hot shell formed around the NS magnetosphere. The accretion rate onto the NS is determined by the ability of the plasma to enter the magnetosphere. Due to large Reynolds numbers in the shell, the interaction of the rotating magnetosphere with plasma initiates a subsonic turbulence. The convective motions are capable of carrying the angular momentum through the shell. We carry out a population synthesis of SyXBs in the Galaxy with account for the spin evolution of magnetized NS. The Galactic number of SyXBs with bright $\left(\mathrm{M}_{v}<1\right)$ low-mass red-giant companion is found to be from $\sim 40$ to 120 , and their birthrate is $\sim 5 \times 10^{-5}-10^{-4} \mathrm{yr}^{-1}$. According to our model, among known SyXBs, Sct X-1 and IRXS J180431.1-273932 are wind-fed accretors. GX 1+4 lies in the transition from the wind-fed SyXBs to SyXBs in which the giants overflow their Roche lobe. The model successfully reproduces very long NS spins (such as in IGR J16358-4724 and 4U 1954+31) without the need to invoke very strong magnetic fields.

8th INTEGRAL Workshop "The Restless Gamma-ray Universe”-Integral2010,

September 27-30, 2010

Dublin Ireland

${ }^{*}$ Speaker. 


\begin{tabular}{lccccc}
\hline \hline SyXB & $P_{\mathrm{S}}(\mathrm{s})$ & $\dot{P}_{\mathrm{s}} / P_{\mathrm{s}}$ & $P_{\text {orb }}(\mathrm{d})$ & $L_{\mathrm{X}}\left(\mathrm{erg} \mathrm{s}^{-1}\right)$ & Sp. Type \\
\hline GX 1+4 & $150^{[6]}$ & $\pm 4 \times 10^{-10}$ & $1161^{[7]}$ & $\sim 10^{37[6]}$ & M5 III \\
4U 1954+31 & $\sim 18300^{[8]}$ & $-1.4 \times 10^{-9[8]}$ & $?$ & $4 \times 10^{32}-10^{35[1]}$ & M4 III ${ }^{[1]}$ \\
4U 1700+24 & $?$ & $?$ & $404^{[9]}$ & $2 \times 10^{32}-10^{34[3]}$ & M2 III ${ }^{[9]}$ \\
Sct X-1 & $113^{[3]}$ & $3.9 \times 10^{-9}[3]$ & $?$ & $2 \times 10^{34[3]}$ & Late K/early M I-III $^{[3]}$ \\
IGR J16194-2810 & $?$ & $?$ & $?$ & $\leq 7 \times 10^{34[10]}$ & M2 III ${ }^{[10]}$ \\
IRXS J180431.1-273932 & $494^{[11]}$ & $?$ & $?$ & $\leq 6 \times 10^{34[11]}$ & M6 III ${ }^{[11]}$ \\
IGR J16358-4724 & $5850^{[12]}$ & $3.1 \times 10^{-8[4]}$ & $?$ & $3 \times 10^{32}-3 \times 10^{36[4]}$ & K-M III $^{[15]}$ \\
IGR J16393-4643 & $912^{[13,14]}$ & $1.0 \times 10^{-11[15]}$ & $50.2^{[15]}$ & $?$ & K-M III $^{[15]}$ \\
2XMM J174016.0-290337 & $626^{[16]}$ & $?$ & $?$ & $\sim 3 \times 10^{34[16]}$ & K1 III ${ }^{[16]}$
\end{tabular}

Table 1: Parameters of observed symbiotic X-ray binaries

\section{Introduction}

Symbiotic X-ray binaries (SyXB) form a growing subclass of X-ray binary stars in which accretion onto a magnetized neutron star (NS) occurs from stellar wind of the companion red giant that does not fill its Roche lobe [1]. All these systems are hard X-ray emitters [2] and are low-mass $\mathrm{X}$-ray binaries, with the possible exception of Sct X-1 [3], for which a high-mass solution also is viable. One of these systems, IGR J16358-4726 may harbour a magnetar [4]. Currently about ten sources are classified as SyXBs or candidate systems (see Table 1).

Remarkable features of SyXBs compared to other low-mass X-ray binaries are their long orbital periods and long NS spin periods. In the catalog of 187 low-mass X-ray binaries [5], the orbital periods of $4 \mathrm{U} 1700+24$ and GX $1+4$ are the longest in all orbital periods observed. With 18300 s spin period, $4 \mathrm{U} 1954+31$ is the slowest accretion powered NS [1]. Therefore, a detailed investigation of SyXB is of immense importance for comprehension of evolution of NS in LMXB.

\section{Quasi-spherical wind accretion}

The cold stellar wind of the red-giant companion is gravitationally captured by NS at the characteristic distance $R_{G}=2 G M /\left(V_{w}^{2}+v_{o r b}^{2}\right)^{2}$ (the Bondi radius). Here $V_{w}$ is the stellar wind velocity at the NS orbital distance, $v_{o r b}$ is the NS orbital velocity. We consider the case of ineffective cooling of plasma behind the shock, so the bow shock is formed at the characteristic radius $\sim R_{G}$. Behind the shock front, the matter falls with a subsonic velocity toward the NS magnetosphere located at a distance $R_{A}$ (the Alfven radius) which is determined by the pressure balance in the accreting matter and the magnetic field. To within a numerical factor of the order of a few, which depends on the magnetosphere structure, $R_{A} \sim\left[\mu^{2} /(\dot{M} \sqrt{2 G M})\right]^{2 / 7}$.

The matter carries the specific angular momentum $j_{w}=k_{w} \Omega_{B} R_{G}^{2}$ (here $-1<k_{w}<0.4$ is the numerical coefficient [17], $\Omega_{B}$ is the binary orbital angular frequency). If $j_{w}>j_{K}\left(R_{A}\right)=\sqrt{G M R_{A}}$, an accretion disc is formed around the NS magnetosphere. In the opposite case, the accretion proceeds quasi-spherically. The interaction of accreting matter with the NS magnetosphere ultimately leads to the NS spin-up or spin-down - the phenomenon that has been intensively studied [18].

The disc accretion on NS magnetospheres is well studied (see [19] for the discussion) and can explain many properties of accreting X-ray pulsars. However, the analysis of spin-down torques in 
a prototypical SyXB GX 1+4 [20,21] shows that the disc accretion can hardly explain the observed correlations.

The quasi-spherical accretion onto slowly rotating magnetized NSs has recently been revised in [22]. In contrast to Bondi accretion, if the cooling is ineffective, the matter can settle onto the NS magnetosphere with a subsonic velocity through a quasi-spherical hot shell. The structure of this shell can be found from the hydrostatic equilibrium: $\rho(R) \sim R^{-3 / 2}, P(R) \sim R^{-5 / 2}, T(R) \sim R^{-1}$. The hot plasma near the shell bottom can be marginally stable against interchange instabilitites mediating plasma entering the magnetosphere, and for accretion to become possible the plasma cooling (e.g. via Compton scattering of X-ray photons) may be required [23]. However, the very fact of observations of slow X-ray pulsars accreting from stellar wind and experiencing spin-down episodes suggests that plasma finds ways to enter the magnetosphere.

In our model the accretion rate onto the NS, which determines its X-ray luminosity, is always smaller than the Bondi-Hoyle accretion rate potentially available by the neutron star $\dot{M}_{G} \sim$ $\rho_{w} R_{G}^{2} / v_{w}^{3}$; the extra matter gravitationally captured by the NS should bend around the hot shell and carry away the angular momentum in the accretion wake.

As shown in [22], the Reynolds number in the hot shell is very high so the interaction of the rotating magnetosphere with the shell initiates subsonic turbulence. At the bottom of the shell the energy is released due to interaction of the shell with the magnetosphere and turbulence, so powerful convective motions begins and establish nearly iso-angular-momentum rotation law in the shell: $\omega(R) \sim R^{-2}$. The NS will spin-up or spin-down depending on the specific angular momentum of matter at the magnetospheric boundary and the angular velocity of the NS rotation $\omega^{*}=2 \pi / P_{s}$. The spin-up/spin-down equation of the quasi-spherically accreting neutron star can be written in the form:

$$
I \dot{\omega}^{*}=Z \dot{M} \omega_{B} R_{G}^{2}-(Z-z) \dot{M} R_{A}^{2} \omega^{*},
$$

where $Z$ and $0<z<1$ are some dimensionless coefficients. Clearly, for spin-down episodes to become possible the condition $Z>z$ must be satisfied. Analysis of torque reversals observed in wind-accreting X-ray pulsars GX 1+4, Vela X-1, GX 302-1 and Cen X-3 shows that in the case of quasi-spherical accretion $Z \gtrsim 1$ in these pulsars [22]. The above equation for the NS spin evolution allows us to estimate the NS magnetic moment of X-ray pulsars in which $\dot{\omega}^{*}$ can be measured as a function of the X-ray flux near the torque reversal point (i.e. where $\dot{\omega}^{*}=0$ ). Remarkably, the estimate of the NS magnetic moment $\mu$ is independent of the actual value of the X-ray luminosity, only $\dot{M}\left(\partial \dot{\omega}^{*} / \partial \dot{M}\right)$ near the torque reversal point matters. The magnetic field of NS in Vela X-1 and GX 301-2 estimated in this way is consistent with the value inferred from the cyclotron line measurements.

The very slow pulsar spins in SyXBs $\left(\omega^{*} R_{A}<\omega_{K}\left(R_{A}\right)\right)$ make it hard to establish the propeller regime to eject matter with parabolic velocities from the magnetosphere during spin-down episodes. The important ratio of viscose tensions to the gas pressure at the magnetospheric boundary is proportional to $\left(\omega^{*}-\omega\left(R_{A}\right)\right) / \omega_{K}\left(R_{A}\right)$, with the maximum value for the non-rotating envelope with $\omega\left(R_{A}\right)=0$. For very slowly rotating pulsars this ratio is very small, i.e. only large-scale convective motions with the characteristic hierarchy of eddies scaled with radius can be established in the shell. When $\omega^{*}>\omega_{K}\left(R_{A}\right)$, the super-sonic propeller regime must set in. In that case the maximum possible spin-down torque is $\sim \mu^{2} / R_{A}^{3}$. It is not excluded that a hot envelope will 


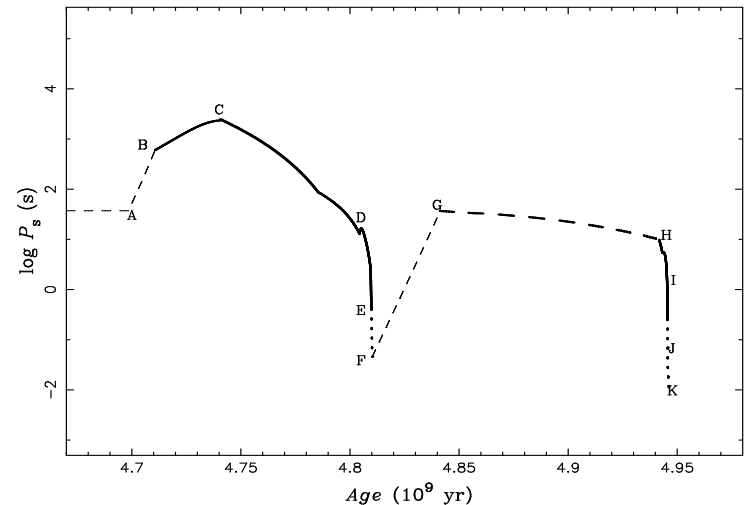

Figure 1: The spin evolution of an accreting magnetized NS in a binary system

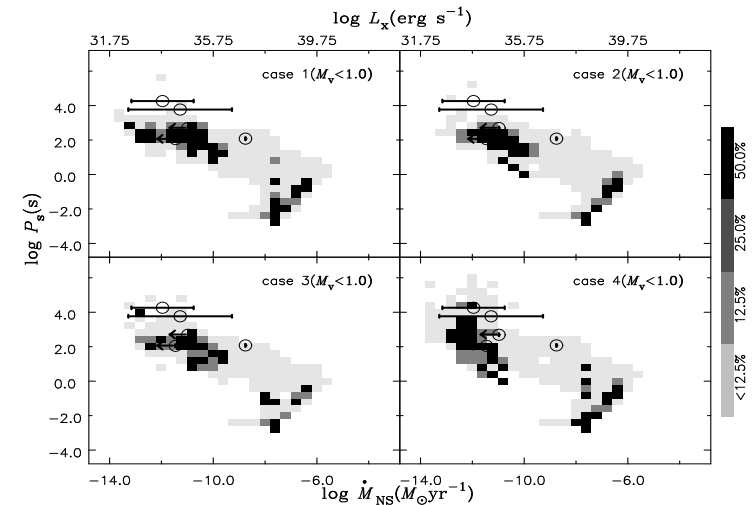

Figure 2: X-ray luminosities and pulsar spin periods in SyXBs with bright red-giants for different models (see [29] for more detail). Open circles show sources from Table 1.

persist here, too, and bring away the angular momentum from the rotating magnetosphere. If the characteristic gas cooling time in the envelope is short, one can expect the formation of a 'storaging' thin Keplerian disc [25]. There is no accretion of matter through such a disc, it only serves to remove the angular momentum from the magnetosphere.

At high $\dot{M}_{G}$, thermal instability can appear in hot plasma behind the bow shock front. The medium can be stratified into cold dense and hot rarefied regions. Cold blobs move in the hot $(\mathscr{R} T \sim G M / R)$ plasma. As they move deeper into the hot shell, their velocity can exceed the local sound velocity, and blobs get gradually destroyed. Crossing the line of sight, these cold blobs would produce an X-ray flux variability with increased hardness ratio. At high X-ray luminosities (high $\dot{M})$ processes of Compton heating and cooling near and above the Alfven radius become important. Below some characteristic distance the Compton cooling by $\mathrm{X}$-rays dominates, and above it the Compton heating prevails. This radius can be determined from the condition $T\left(R_{x}\right) \simeq T_{X}$, where $T_{X}$ is the effective temperature of X-ray photons. The latter depends on the spectral shape (for example, $T_{X}=1 / 4 T$ for spectrum with an exponential cutoff $\simeq e^{-E / k T}, T_{X}=T$ for the Planckian spectrum). If the Compton cooling time is shorter than the fall time of matter, non-stationary accretion regime sets in [26]. Real wind-accreting systems demonstrate complex quasi-stationary behavior with dips, outbursts, etc. These features can be interpreted using the proposed model of quasi-spherical accretion.

\section{Population synthesis of SyXB}

For the simulation of binary evolution, we use the rapid binary star evolution code BSE [27] with updates by [28]. The code is appended by an algorithm for the careful treatment of spin evolution of a magnetized NS and the NS magnetic field decay, as described in [29]. The important parameter for the SyXB evolution is the stellar wind velocity from the red giant, which we parametrize as $v_{w}=\sqrt{\beta(2 G M / R)}$. The parameter $\beta$ is set to 1 .

An example of the spin evolution of a magnetized NS in SyXB is presented in Fig. 1. The parameter for the specific angular momentum of captured stellar wind is $k_{w}=1 / 4$. We start with 
a binary consisting of a $1.4 M_{\odot}$ NS with the surface magnetic field $5 \times 10^{12} \mathrm{G}$, the spin period $P_{s}=0.01 \mathrm{~s}$, and a $1.3 M_{\odot}$ main-sequence star. The initial orbital period of the system is $P_{B}=400 \mathrm{~d}$. Before point 'A', the secondary is still a MS-star and $\dot{M}_{G}$ onto the NS is below $10^{-15} M_{\odot} \mathrm{yr}^{-1}$, so the NS evolves like an isolated object. The evolution of $P_{\mathrm{s}}$ is determined by the spin-down torque calculated by the magneto-dipole formula. At point ' $A$ ' the secondary enters the Hertzsprung gap and starts to lose mass via stellar wind. The Bondi radius is $R_{\mathrm{G}}=1.3 \times 10^{13} \mathrm{~cm}$, relations $R_{G}>$ $R_{A}>R_{c}=\left(G M / \omega^{* 2}\right)^{1 / 3}$ and $j_{w}<j_{K}\left(R_{A}\right)$ hold, so the NS enters the super-sonic propeller stage of quasi-spherical accretion. The NS spin period (and hence the corotation radius $R_{c}$ ) increases, and at point 'B' $R_{A}=R_{c}$. The NS turns out at the quasi-spherical accretor state from stellar wind, and the evolution of $P_{s}$ is determined by Eq. 2.1. The SyXB stage starts. At point 'C', the secondary evolves to the first giant branch (FGB) stage. The NS is still in the accretor state. With the ascend along FGB, the stellar wind velocity of the giant $v_{w}$ decreases so that $j_{w} \sim v_{w}^{-4}$ increases. At point 'D', $j_{w}>j_{K}\left(R_{A}\right)$, and a wind-fed accretion disc forms around the NS. At point 'E' the secondary overfills its Roche lobe, the mass accretion rate immediately increases and exceeds the critical Eddington rate. The NS becomes a super-accretor. At point 'F', the secondary leaves FGB and evolves to the core helium burning stage. The mass-loss rate rapidly decreases, but the velocity of the stellar wind is low so that $j_{w}>j_{K}\left(R_{A}\right)$ and the NS becomes a propeller with a wind-fed accretion disc. At point ' $G$ ', the secondary evolves to early asymptotic giant branch (E-AGB). At point ' $\mathrm{H}^{\prime} R_{A} \leq R_{c}$ and the NS becomes a wind-fed accretor again. For a short time the system again appears as a SyXB. Due to the high mass accretion rate, the spin period $P_{\mathrm{S}}$ rapidly decreases. At point ' $I$ ' the secondary overfills its Roche lobe again, so that the supercritical disc is formed. At point ' $\mathrm{J}$ ' the secondary evolves into thermally pulsing AGB-star. At point ' $\mathrm{K}$ ' the secondary leaves the AGB to become a hot white dwarf. To summarize, between points ' $\mathrm{B}$ ' and ' $\mathrm{E}$ ' and from ' $\mathrm{H}$ ' to ' $\mathrm{K}$ ' the considered system appears as a SyXB. Note that the torque reversal from spin-down to spin-up occurred close to point ' $\mathrm{C}$ ' when the mass loss rate from the red giant gradually increased, in accordance with Eq. 2.1.

\section{Results and conclusion}

We have used the treatment of quasi-spherical wind accretion considered in [22] to calculate the properties of a population of SyXB in the Galaxy. The resulting population of SyXBs on the $P_{s}-L_{x}$ plane is shown in Fig. 2, in which known SyXBs from Table 1 are also plotted by open circles. Several population synthesis models (with different kick velocities of the nascent NS and stellar wind parameters) were calculated (see [29] for more detail). The main results are:

1. The galactic birthrate of all SyXBs is $\sim(4-9) \times 10^{-5} \mathrm{yr}^{-1}$. The total number of galactic SyXBs is between $\sim 5000$ and $\sim 10000$. The birthrate of SyXB mostly depends on the kick velocity dispersion imparted to nascent NS in core-collapse supernovae. However, this parameter affects the population of SyXB by a factor of about 2 .

2. $70 \%-80 \%$ of NSs in SyXB are formed in core-collapse supernovae, $10 \%-20 \%$ are formed in electron-capture supernovae. Less than $\sim 1 \%$ of SyXBs experience super-Eddington accretion.

3. The galactic number of SyXBs with bright (the absolute stellar magnitude $M_{v}<1$ ) low-mass red-giant companion are from $\sim 40$ to 120 , and their birthrate is $\sim(5-8) \times 10^{-5} \mathrm{yr}^{-1}$. According 
to our model, among known SyXBs, Sct X-1 and IRXS J180431.1-273932 are wind-fed accretors. GX 1+4 lies in the transition from the wind-fed SyXBs to SyXBs in which the giants overflow their Roche lobe. The model successfully reproduces very long NS spins (such as in IGR J16358-4724 and $4 \mathrm{U} 1954+31$ ) without the need to invoke very strong magnetic fields.

\section{References}

[1] N. Masetti, M. Orlandini, E. Palazzi, L. Amati and F. Frontera, A\&A 453295 (2006).

[2] U. Mürset, B. Woolff and S. Jordan, A\&A 474 L1 (1997).

[3] D.L. Kaplan, A.M. Levine, D. Chakrabarty et al., ApJ 661437 (2007).

[4] S.K. Patel, J. Zurita, M. Del Santo et al., ApJ 657994 (2007).

[5] Q.Z. Liu, J. van Paradijs and E.P.J. van den Heuvel, $A \& A 469807$ (2007).

[6] D. Chakrabarty and P. Roche, ApJ 489254 (1997).

[7] K.H. Hinkle, F.C. Fekel, R.R. Joyce, P.R. Wood, V.V. Smith and T. Lebzelter, ApJ 641479 (2006).

[8] R.H.D. Corbet, J.L. Sokoloski, K. Mukai, C.B. Markwardt, J. Tueller, ApJ 675424 (2008).

[9] N. Masetti, D. Dal Fiume, G. Cusumano et al., A\&A 382104 (2002).

[10] N. Masetti, R. Landi, M.L. Pretorius et al., A\&A 470331 (2007).

[11] A.A. Nucita, S. Carpano and M. Guainazzi, A\&A 474 L1 (2007).

[12] S.K. Patel, C. Kouveliotou, A. Tennant et al., ApJ 602 L45 (2004).

[13] A. Bodaghee, R. Walter R., J.A. Zurita Heras et al., A\&A 4471027 (2006).

[14] T.W.J. Thompson, J.A. Tomsick, R.E. Rothschild, J.J.M. in't Zand and R. Walter, ApJ 649373 (2006).

[15] E. Nespoli, J. Fabregat and R.E. Mennickent, A\&A 516 idA94 (2010) [arXiv:0910.0990].

[16] S.A. Farrell, A.J. Gosling, N.A. Webb, D. Barret, S.R. Rosen, M. Sakano, B. Pancrazi, A\&A $\mathbf{5 2 3}$ idA50 (2010) [arXiv:1008.4352].

[17] C. Ho, R.E. Taam, B.A. Fryxell, T. Matsuda and H. Koide, MNRAS 2381447 (1989).

[18] L. Bildsten L., D. Chakrabarty, J. Chiu et al., ApJS 113367 (1997).

[19] R.V.E. Lovelace, M.M. Romanova and G.S. Bisnovatyi-Kogan, MNRAS 275244 (1995).

[20] D. Chakrabarty, L. Bildsten, M.H. Finger et al., ApJ 481 L101 (1997).

[21] A. González-Galán, E. Kuulkers, P. Kretschmar, S. Larsson, K. Postnov, A. Kochetkova, and M. H. Finger, Spin period evolution of $G X 1+4$, in prep.

[22] N.I. Shakura, K. A. Postnov, D. K. Klochkov, V. Doroshenko, V. Suleimanov, and L. Hjalmarsdotter, 2011, Accretion torques in wind-accreting pulsars, in prep.

[23] J. Arons and S. Lea, ApJ 207914 (1976).

[24] M.E. Davies and J.E. Pringle, MNRAS 196209 (1981).

[25] N.I. Shakura and R.A. Sunyaev, MNRAS 175613 (1976).

[26] R.A. Sunyaev, In: Physics and astrophysics of neutron stars and black holes. Amsterdam, North Holland Publishing Co., p. 697 (1978). 
[27] J.R. Hurley, C.A. Tout and O. Pols, MNRAS 329897 (2002).

[28] P.D. Kiel and J.R. Hurley, MNRAS 3691152 (2006).

[29] G.-L. Lü, C.-H. Zhu, K. A. Postnov, L. Yungelson, A. G. Kuranov and N. Wang, Population Synthesis for Symbiotic X-ray Binaries, in prep. 